\title{
An increase in switching frequency correlates with an increase in recombination of the ribosomal chromosomes of Candida albicans strain 3153A
}

\author{
H. Ramsey, B. Morrow and D. R. Soll \\ Author for correspondence: David R. Soll. Tel: +1 319335 1117. Fax: +1 3193352772.
}

Department of Biological Sciences, University of lowa, lowa City, lowa 52242, USA

\begin{abstract}
When cells of Candida albicans strain 3153A are treated with a low dose of ultraviolet irradiation, they switch at high frequency $\left(10^{-2}\right)$ between a number of switch phenotypes discriminated by colony morphology. Clones from switching lineages exhibit continuous reorganization of their ribosomal chromosomes ( $R$ chromosomes), while clones from lineages maintaining the original smooth phenotype exhibit no reorganization. $R$ chromosome reorganization results in decreases as well as increases in size of the chromosomes, but changes are not reciprocal between $\mathbf{R}$ chromosome homologues.
\end{abstract}

Keywords: phenotypic switching, ribosomal chromosome reorganization, Candida albicans

\section{INTRODUCTION}

Candida albicans and related species are capable of switching at extremely high frequencies between a number of phenotypes distinguished by colony morphology (Pomes et al., 1985; Slutsky et al., 1985, 1987; Soll, 1992; Soll et al., 1993a). Strains can differ in the variant phenotypes in their switching repertoire, but switching systems share several general characteristics (Soll, 1992) and, in the case of two tested switching systems, involve the regulation of the same phase-specific genes (Morrow et al., 1992, 1994). Recently, it was demonstrated that strains of $C$. albicans exhibit a high degree of karyotype polymorphism which can be visualized by chromosome separation techniques such as transverse alternating field electrophoresis (TAFE), orthogonal field alternating gel electrophoresis (OFAGE) and contour clamped homogeneous electric field electrophoresis (CHEFE) (Magee \& Magee, 1987; Merz et al., 1988; Lasker et al., 1989; Suzuki et al., 1989; Rustchenko-Bulgac et al., 1990; Rustchenko-Bulgac, 1991). These changes have been observed over time in single strains (Suzuki et al., 1989; Rustchenko-Bulgac et al., 1990; Rustchenko-Bulgac, 1991), and occur more frequently in the chromosomal homologues containing

Abbreviations: TAFE, transverse alternating field elecrophoresis; osmooth, original smooth; R chromosome, ribosomal chromosome; R1, high- $M_{r} R$ chromosome homologue; $R 2$, low- $M, R$ chromosome homologue. ribosomal cistrons ( $\mathrm{R}$ chromosomes) (Suzuki et al., 1989; Rustchenko-Bulgac, 1991).

In a recent analysis of morphological variants of $C$. albicans strain $3153 \mathrm{~A}$, karyotype variability was observed and interpreted to reflect a relationship between high frequency phenotypic switching and chromosomal reorganization (Rustchenko-Bulgac et al., 1990; Rustchenko-Bulgac, 1991; Soll, 1992). However, karyotype stability has been demonstrated for the 'white-opaque' switching system in C. albicans strain WO-1 (Rikkerink et al., 1988; McEachern \& Hicks, 1991; Soll, 1992), suggesting that if there is a relationship between changes in the size of chromosomes and switching, it is strainspecific and most likely reflects a parallel increase in the frequencies of the two processes rather than a cause-effect relationship between them (Soll, 1992). In addition, in the studies correlating phenotypic variants with karyotypic changes in strain 3153A (Rustchenko-Bulgac et al., 1990; Rustchenko-Bulgac, 1991), experiments to test whether karyotypic changes occurred at high frequency in the absence of phenotypic switching were not reported.

To investigate the possible relationship between karyotypic change and switching in strain 3153A, we have compared individual switching lineages of variant phenotypes, which are in a high frequency mode of switching, with parallel control lineages of cells showing the basic original smooth (o-smooth) phenotype, which are in a low frequency mode of switching. Our results demonstrate that, in strain 3153A, control cells in a low frequency 
mode of switching do not undergo chromosomal reorganization through clonal lineages while cells exhibiting a high frequency mode of switching undergo continuous reorganization of the $\mathrm{R}$ chromosomes. Reorganization results in both decreases and increases in the sizes of both $\mathrm{R}$ chromosomes, but the changes are not reciprocal.

\section{METHODS}

Maintenance of stock cultures of the basic 0-smooth phenotype. Cells from a storage slant of $C$. albicans strain 3153A were plated on agar containing the nutrient composition of Lee's amino-acid-rich, defined medium (Lee et al., 1975) supplemented with $70 \mu \mathrm{g}$ arginine $\mathrm{ml}^{-1}$ and $0 \cdot 1 \mu \mathrm{M} \mathrm{ZnSO}_{4}$ (referred to as modified Lee's medium; Bedell \& Soll, 1979). After 7-9 d incubation at $25^{\circ} \mathrm{C}$, cells from a single o-smooth colony were diluted into distilled water and plated on fresh agar at a density of 50-70 colony forming units (c.f.u.) per plate. For the unirradiated o-smooth lineage, cells from a representative clone in the second set of agar plates were suspended in distilled water and plated again on agar at 50-70 c.f.u. per plate. In addition, cells from the same representative colony were inoculated into modified Lee's liquid medium, grown to late exponential phase in suspension at $25^{\circ} \mathrm{C}$, and then processed for TAFE.

Induction of high frequency switching and genesis of switching lineages. Cells from an o-smooth colony were grown in modified Lee's liquid medium at $25^{\circ} \mathrm{C}$ to the mid-exponential phase of growth. Cells were then irradiated for $5 \mathrm{~s}$ with UV light (total energy output $31 \mathrm{~J} \mathrm{~m}^{-\mathbf{2}}$ ) according to the methods of Morrow et al. (1989), then diluted and spread on agar plates containing modified Lee's medium. Cells were plated at $40-70$ c.f.u. per plate. After $7 \mathrm{~d}$ incubation at $25^{\circ} \mathrm{C}$, an irregular wrinkle colony was selected, and cells from this colony were diluted and plated to initiate switching lineages. Several smooth colonies were also selected and plated. One colony which was still in a low frequency mode of switching was selected to generate a UV-treated, non-switching lineage.

Pulsed field electrophoresis. Cells were grown to mid-exponential phase in modified Lee's liquid medium at $25^{\circ} \mathrm{C}$ and embedded in agarose plugs according to the methods of Vollrath \& Davis (1987). Chromosome-sized DNA molecules were separated in a $0.65 \%$ agarose gel in buffer containing $10 \mathrm{mM}$ Tris/ $\mathrm{HCl}, 4.35 \mathrm{mM}$ acetic acid and $0.5 \mathrm{mM}$ EDTA, using TAFE (Geneline, Beckman Instruments). Gels were run at $100 \mathrm{~V}$ with the following pulse regime: 1 min pulses for $6 \mathrm{~h}$, $2 \mathrm{~min}$ pulses for $12 \mathrm{~h}, 4 \mathrm{~min}$ pulses for $16 \mathrm{~h}$, and $7 \mathrm{~min}$ pulses for $20 \mathrm{~h}$. This was followed by $10 \mathrm{~min}$ pulses at $80 \mathrm{~V}$ for $18 \mathrm{~h}$. Gels were then stained with a $0.032 \%$ aqueous solution of ethidium bromide.

Southern blot analysis of TAFE gels. TAFE gels were blotted onto Zetabind filters (Cuno) (Morrow et al., 1992). DNA was cross-linked to the filter by UV irradiation and hybridized at $65^{\circ} \mathrm{C}$ with a nick-translated ribosomal DNA probe of $\mathrm{C}$. albicans. The ribosomal probe was a $3.4 \mathrm{~kb}$ EcoRI fragment cloned from a genomic library. Nucleotide sequence analysis revealed that this fragment contains the coding sequence for both the $25 \mathrm{~S}$ and $5.8 \mathrm{~S}$ rRNAs (T. Srikantha, R. R. Gutell, B. Morrow \& D. R. Soll, unpublished). Hybridized blots were then pressed against XAR-S film (Eastman Kodak) with a Cronex Lightning-Plus intensifying screen (DuPont) in a lightproof chamber.

DNA fingerprinting. DNA fingerprinting was performed according to the methods of Schmid et al. (1990) with the moderately repetitive sequence Ca3 (Sadhu et al., 1991; Anderson et al., 1993; Soll, 1993).

\section{RESULTS}

\section{Switching lineages and switching frequencies of strain 3153A}

Cells plated from storage cultures of strain 3153A exhibited the basic o-smooth colony morphology (Fig. 1a) on the zinc-limiting agar containing the nutrient components of modified Lee's medium (see Methods), which has been employed to visualize the variant phenotypes in the $3153 \mathrm{~A}$ switching repertoire (Slutsky et al., 1985; Soll, 1992). Cells in the o-smooth phenotype were previously demonstrated to switch spontaneously to variant phenotypes at frequencies of roughly $10^{-4}$ (Slutsky et al., 1985). In the present study, no spontaneous variant colonies were observed in 5832 colonies plated from osmooth stock cultures. Therefore, the spontaneous
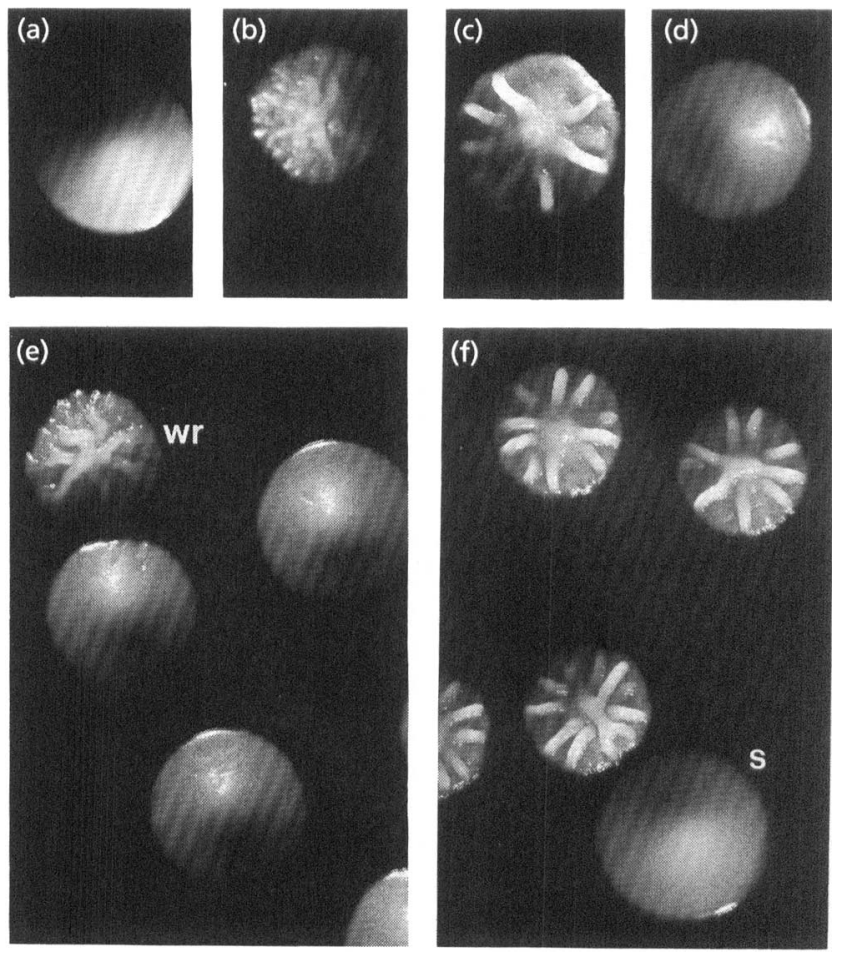

Fig. 1. Switching in Candida albicans strain 3153A. To initiate high frequency switching, a stock culture was treated with a low dose of UV irradiation, killing less than $8 \%$ of the cell population. (a) Original smooth (o-smooth) colony morphology exhibited by cells from the stock culture. (b) Irregular wrinkle colony morphology. (c) Star colony morphology. (d) Revertant smooth ( $r$-smooth) colony morphology (revertant smooth is slightly less smooth than o-smooth and still in a high frequency mode of switching). (e) Example of cells plated from an $r$-smooth colony in which one colony (wr) has switched to irregular wrinkle phenotype. (f) Example of cells plated from a star colony in which one colony (s) has switched to a smooth phenotype. In all cases, colonies were photographed after growth at $25^{\circ} \mathrm{C}$ for 7-9 d. 
Table 1. Frequency of variant colony formation in the switching and nonswitching lineages analysed

\begin{tabular}{|c|c|c|c|}
\hline $\begin{array}{l}\text { Switching } \\
\text { lineage }\end{array}$ & $\begin{array}{c}\text { No. of } \\
\text { colones } \\
\text { plated }\end{array}$ & $\begin{array}{c}\% \\
\text { Original } \\
\text { phenotype }\end{array}$ & $\begin{array}{c}\text { Frequency of } \\
\text { switching }\end{array}$ \\
\hline \multicolumn{4}{|l|}{ (a) Lineage 1} \\
\hline O-smooth & 1500 & 100 & $<6.7 \times 10^{4 *} \leftarrow U V \dagger$ \\
\hline Irr. wrinkle & 1568 & 11 & $\mathrm{MC} \ddagger$ \\
\hline Rev. smooth $\$$ & 3727 & $99 \cdot 8$ & $2 \cdot 1 \times 10^{-3}$ \\
\hline Irr. wrinkle & 9543 & $0 \cdot 1$ & $\mathrm{MC} \ddagger$ \\
\hline Star & 2601 & $99 \cdot 6$ & $4 \cdot 2 \times 10^{-3}$ \\
\hline Rev. smooth & 2057 & $99 \cdot 8$ & $1.9 \times 10^{-3}$ \\
\hline Star & 1149 & $86 \cdot 1$ & $1.6 \times 10^{-1}$ \\
\hline Rev. smooth\| & - & - & - \\
\hline \multicolumn{4}{|l|}{ (b) Lineage 2} \\
\hline O-smooth & 1500 & 100 & $<6.7 \times 10^{-4} \leftarrow \mathrm{UV} \uparrow$ \\
\hline Irr. wrinkle & 1568 & 11 & MC \\
\hline Star & 3140 & 58 & $4 \cdot 2 \times 10^{-1}$ \\
\hline Rev. smooth & 1431 & $99 \cdot 7$ & $2.7 \times 10^{-3}$ \\
\hline Star & 1516 & $99 \cdot 5$ & $5 \cdot 3 \times 10^{-3}$ \\
\hline Rev. smooth $\|$ & - & - & - \\
\hline \multicolumn{4}{|l|}{ (c) Lineage 3} \\
\hline O-smooth & 1813 & 100 & $<5.5 \times 10^{-4}$ \\
\hline O-smooth & 635 & 100 & $<1.5 \times 10^{-3}$ \\
\hline O-smooth & 1003 & 100 & $<9.9 \times 10^{-4}$ \\
\hline O-smooth & 881 & 100 & $<1 \cdot 1 \times 10^{-3}$ \\
\hline \multicolumn{4}{|l|}{ (d) Lineage 4} \\
\hline O-smooth & 1500 & 100 & $<6.7 \times 10^{-4} \leftarrow \mathrm{UV} \dagger$ \\
\hline O-smooth & 1438 & 100 & $<7.0 \times 10^{-4}$ \\
\hline O-smooth & 1137 & 100 & $<8.8 \times 10^{-4}$ \\
\hline O-smooth & 1125 & 100 & $<8.9 \times 10^{-4}$ \\
\hline O-smooth & 687 & 100 & $<1.5 \times 10^{-3}$ \\
\hline
\end{tabular}

* Since no colonies out of 1500 were variant, the estimated frequency had to be below 1 in 1500 or less than $6.7 \times 10^{4}$.

†UV treatment (see Methods).

$\ddagger$ In two cases, cells plated from irregular wrinkle colonies in a lineage formed predominantly smooth phenotypes ( $89 \%$ or more) suggesting mass conversion (MC).

$\S$ The plug of revertant smooth was inadvertantly frozen and, therefore, was not karyotyped.

$\|$ The last switch phenotype in each lineage was not plated.

switching frequency of the stock culture of o-smooth cells was below $1.7 \times 10^{-4}$.

It was previously demonstrated that low doses of UV irradiation, which kill less than $15 \%$ of the cell population, stimulate the frequency of switching in strains 3153A (Slutsky et al., 1985), WO-1 (Morrow et al., 1989) and 1001 (Pomes et al., 1985). We therefore irradiated a stock culture of strain 3153A with a low dose of UV, killing $8 \%$ of the cell population. Variant colonies in the surviving population appeared at a frequency of $10^{-2}$, which was at least two orders of magnitude higher than the frequency of switching of an unirradiated cell population. An irregular wrinkle colony (Fig. 1b, e) was selected and the cells in the colony plated. Of 1568 colonies, 1398 exhibited a smooth phenotype, $169(11 \%)$ exhibited the irregular wrinkle phenotype of the parent colony, and 1 exhibited the star phenotype (Fig. 1c, Table $1 \mathrm{a}, \mathrm{b})$. Two independent lineages were initiated from this plating (lineages 1 and 2 in Table $1 \mathrm{a}$ and $\mathrm{b}$, respectively). Lineage 1 included the following sequence of phenotypes, beginning with the original smooth culture: o-smooth, irregular wrinkle, revertant smooth (Fig. 1d; see description of this phenotype in legend to Fig. 1d), irregular wrinkle, star, revertant smooth, star and revertant smooth. Lineage 2 included the following sequence of phenotypes: o-smooth, irregular wrinkle, star, revertant smooth, star and revertant smooth. The frequencies of phenotypes other than the one plated at each step in the two respective switching lineages are presented in Table 1(a) and (b). All phenotypes following o-smooth in the switching lineages exhibited high frequencies of switching. In the two cases of irregular wrinkle, there was mass conversion to a smooth phenotype (Table 1a, b). An example of a switch from revertant smooth to irregular wrinkle is shown in Fig. 1e, and a switch from star to revertant smooth in Fig. 1f.

\section{Karyotypic changes associated with switching lineages}

TAFE separated the chromosomes of cells in the osmooth phenotype into nine bands (o sm, Fig. 2a). A Southern blot of the TAFE gel was hybridized with a nick-translated ribosomal probe. The two highest $M_{\mathrm{r}}$ bands hybridized with the ribosomal probe. Since $C$. albicans is diploid, the two bands were considered to contain unequal-sized chromosomal homologues carrying ribosomal cistrons (o sm, Fig. 2b). The high- and low- $M_{\mathrm{r}}$ homologues will be referred to as R1 and R2, respectively. TAFE separation of the chromosomes of the switch phenotypes in lineage 1 (Table 1a) demonstrated size changes in both R1 and R2 (Fig. 2a). In the gel pattern of the first irregular wrinkle phenotype in the lineage, a tenth band appeared between the two original, highest $M_{\mathrm{r}}$ bands (i wr, Fig. 2a). Hybridization with the ribosomal probe demonstrated that the size of R1 had decreased dramatically (Fig. 2b), and that R1 represented the new band in the gel pattern, while the size of R2 remained relatively constant (Fig. $2 \mathrm{~b}$ ). Therefore, the decrease in the size of R1 accompanying the switch from o-smooth to irregular wrinkle did not involve a reciprocal increase in the size of R2. Since the highest $M_{\mathrm{r}}$ band in the ethidiumbromide-stained o-smooth gel pattern was also present in the irregular wrinkle gel pattern (Fig. 2a), but no longer hybridized to the ribosomal probe (Fig. 2b), we can conclude that this band contained non-homologous chromosomes of roughly equal size, one of which was R1, and that only R1 changed size in the switch to irregular wrinkle in the first step in this lineage. 
(a)

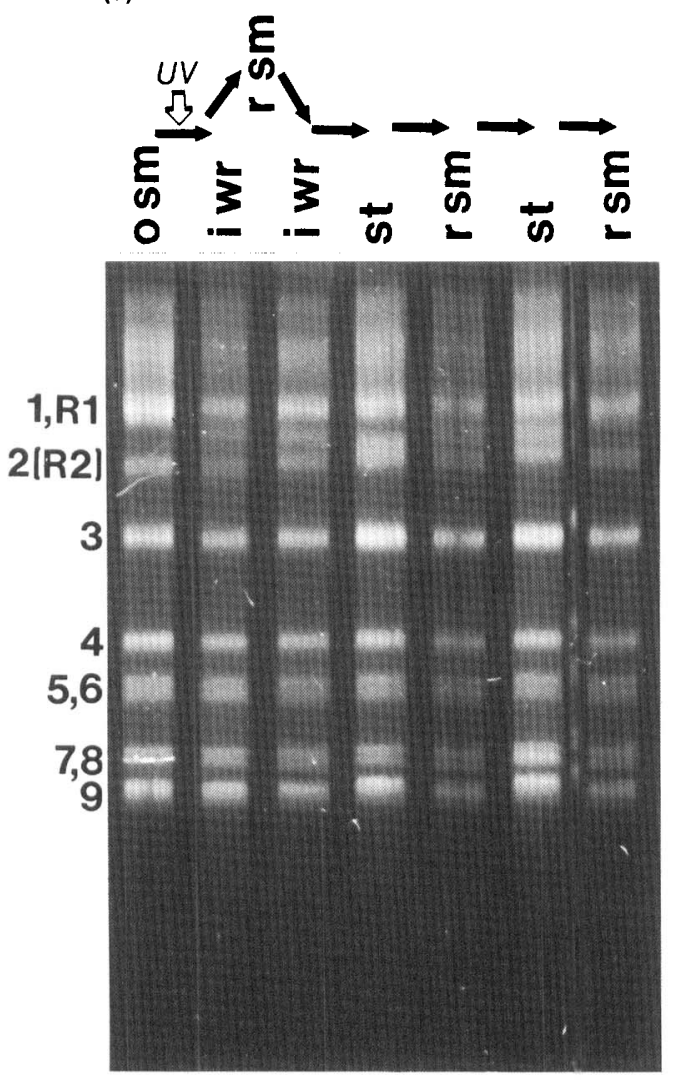

(b)

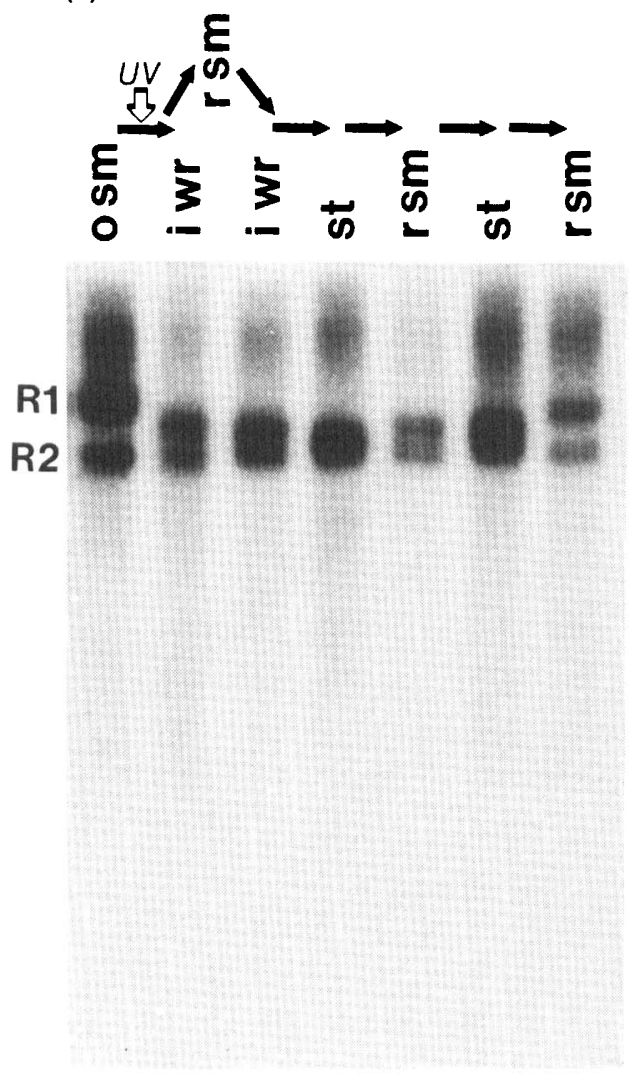

Fig. 2. Changes in the size of $R$ chromosomes through a switching lineage. O-smooth cells were treated with a low dose of UV irradiation, and a sequence of variant phenotypes were clonally isolated and replated. This represents switching lineage 1 in Table 1(a). Cells from each phenotype in the switching lineage were grown in liquid medium and then prepared for chromosome separation by TAFE. The phenotypes are in the lineage connected by arrows at the top of each panel. (a) Ethidium bromide-stained TAFE gel of separated chromosomes. (b) Southern blot hybridization of the TAFE gel with a labelled ribosomal probe. In this particular lineage, the third isolate, $r$-smooth, was not fingerprinted. 0 sm, original smooth; i wr, irregular wrinkle; $r$ sm, revertant smooth; st, star. 1 through 9 to the left of (a) represent band numbers; R1 and R2 represent the larger and smaller chromosomal homologues containing ribosomal cistrons. Note that band 1 contains two chromosomes, one of which is an R chromosome. Band 2 contains only an R chromosome.

In the next three steps of switching in lineage 1 (irregular wrinkle to revertant smooth to irregular wrinkle to star), $\mathrm{R} 1$ continued to decrease in size, approaching the size of R2 (Fig. 2a, b). However, in the next three switches (star to revertant smooth to star to revertant smooth), R1 increased in size (Fig. 2a, b). At the end of lineage 1, one of the homologues, presumably R1, had returned to its original size. After the first switch, the size of $\mathrm{R} 2$ increased steadily. This is evident when the gap between R 2 and the third highest $M_{\mathrm{r}}$ band is followed through the lineage in the ethidium-bromide-stained gel pattern of switching lineage 1 (Fig. 2a).

In a second switching lineage (lineage 2 in Table 1) emanating from the same UV-induced irregular wrinkle colony as lineage 1 , the sizes of both $\mathrm{R} 1$ and $\mathrm{R} 2$ changed, but the changes did not follow the scenario of lineage 1 , and the changes in R1 and R2 were again not reciprocal. Through five sequential switches (o-smooth to irregular wrinkle to star to revertant smooth to star to revertant smooth), the size of R1 continued to decrease (Fig. 3). As in the first lineage, the size of R2 remained constant through the first switch, but then decreased continuously through the four subsequent switches (Fig. 3). This contrasts with the scenario of lineage 1 , in which the size of R1 decreased and then increased, and the size of R2 increased continuously after the first switch.

\section{Karyotype stability in the absence of switching}

To determine whether the high frequency of reorganization of $\mathrm{R}$ chromosomes is an exclusive characteristic of cells in a high frequency mode of switching, or a characteristic of strain 3153A regardless of switching frequency, sequential clones were karyotyped in two lineages (lineages 3 and 4 in Table 1) in which cells remained in the o-smooth phenotype in a low frequency mode of switching. The two nonswitching lineages differed. In the first (lineage 3 in Table 1), o-smooth clones were sequentially isolated without initial UV irradiation. In the second (lineage 4 in Table 1), o-smooth cells were 


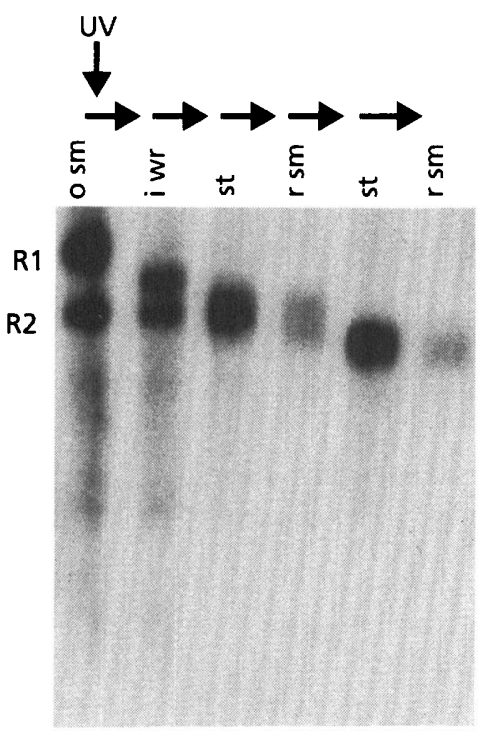

Fig. 3. Changes in the sizes of the $R$ homologues in a second switching lineage. Cells from each step in the lineage, described at the top of the gel, were grown in liquid medium. Chromosomes were then separated by TAFE, and the Southern blot was hybridized with a radiolabelled ribosomal probe. $O$ sm, o-smooth; i wr, irregular wrinkle, st, star; $r$ sm, revertant smooth. Note that in this lineage, R1 continually decreased in size; $R 2$ remained constant through the first star phenotype, then decreased in size.

treated with UV, and an o-smooth colony was selected from the post-irradiated culture which remained in a low frequency mode of switching throughout the subsequent o-smooth lineage. The frequency of spontaneous switching was below $1 \cdot 1 \times 10^{-3}$ for the sequential clones in lineage 3 (Table $1 \mathrm{c}$ ), and below $1.5 \times 10^{-3}$ for sequential clones in lineage 4 (Table 1d).

TAFE separation of chromosomes was performed on sequential isolates in the same manner as that for switching lineages 1 and 2 . In both nonswitching lineages, the ethidium-bromide-stained gel patterns remained constant (Fig. 4a, b). No change was observed in the size of R1, R2 or any other chromosomal band.

\section{Variants in the switching lineages are not storage contaminants}

In the isolation of colonies with variant morphologies 7-10 d after plating, the possibility exists that rare variants may actually represent unrelated contaminants. To demonstrate that the changes observed in switching lineages had really occurred in sequential clones of the same strain, the o-smooth isolate prior to UV irradiation, the first irregular wrinkle isolate after UV irradiation, the last star isolate and the last revertant smooth isolate from lineage 1 (Table 1a) were fingerprinted with the moderately repetitive DNA sequence $\mathrm{Ca} 3$, which discriminates between more than $99 \%$ of independently isolated strains of

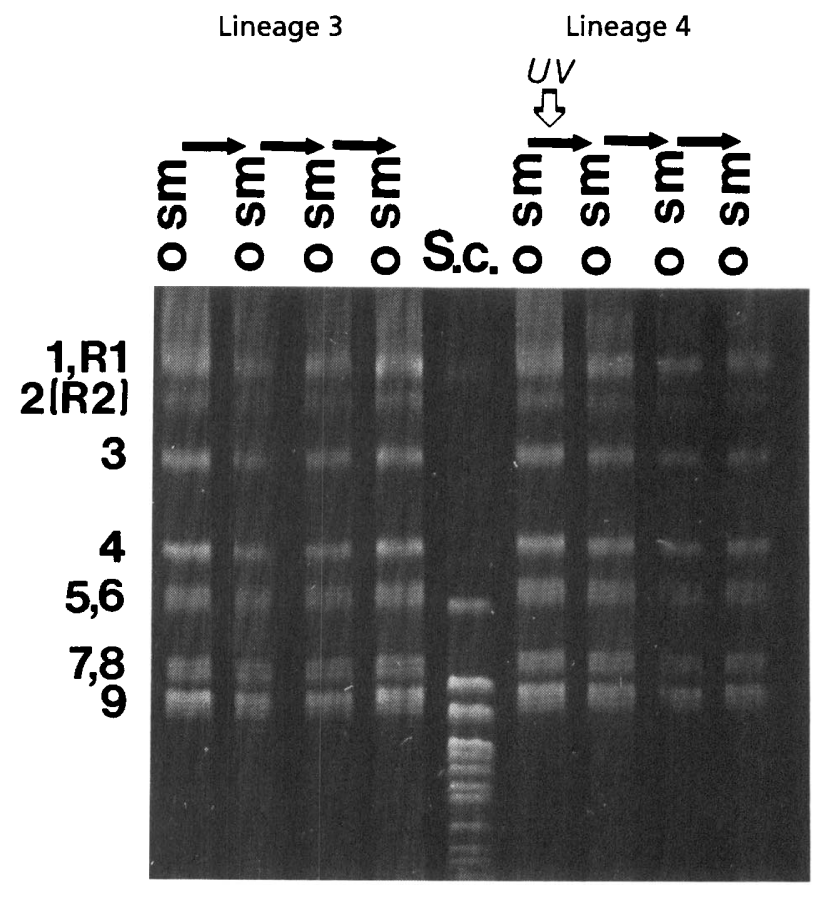

Fig. 4. The constancy of $R$ chromosome size in nonswitching lineages. In lineage 3 (see Table 1c), sequential clones of osmooth were grown in liquid medium and then prepared for chromosome separation by TAFE. In lineage 4 (Table 1d), an osmooth colony, a UV-treated o-smooth progeny clone, and two sequential o-smooth clones emanating from the UV-treated clone were grown in liquid medium and prepared for TAFE. The TAFE gel was stained with ethidium bromide. Note that there are no detectable changes in the size of the $R$ chromosomes through these lineages. S.c., Saccharomyces cerevisiae. Bands are numbered to the left of the gel.

C. albicans (Schmid et al., 1990). The fingerprints of the four isolates were identical (data not shown), demonstrating that variants in the lineage were derived from strain 3153A.

\section{DISCUSSION}

When Rustchenko-Bulgac and co-workers (RustchenkoBulgac et al., 1990; Rustchenko-Bulgac, 1991) reported that morphological variants of strain $3153 \mathrm{~A}$ exhibited chromosomal variations, we assumed that the high frequency of chromosomal variability was either a trait of the strain in general and had nothing to do with switching, or that it was one more phenotypic characteristic of switching, which has been demonstrated to be extremely pleiotropic (Soll, 1990, 1992; Soll et al., 1993a, b). Our conclusion that a change in chromosome size was not the mechanism of switching (Soll, 1992; Soll et al., 1993b) was based on a number of observations. First, three independent laboratories working on strain WO-1 found no variation in the electrophoretic karyotype of cells switching back and forth between the white and opaque phenotypes (Rikkerink et al., 1988; McEachern \& Hicks, 1991; Morrow et al., 1992; Soll, 1992). Second, specific 
changes in karyotype were not demonstrated to correlate with specific variant phenotypes in the 3153A switching system (Rustchenko-Bulgac et al., 1990; RustchenkoBulgac, 1991). Third, the corresponding stability of the electrophoretic karyotype in sequentially isolated nonswitching clones of strain $3153 \mathrm{~A}$ was never demonstrated (Rustchenko-Bulgac et al., 1990; Rustchenko-Bulgac, 1991). We therefore re-examined the possible relationship between electrophoretic karyotype instability and switching in strain 3153A by monitoring the karyotypes of sequential clones in switching lineages, and compared karyotype stability in these lineages with that of sequential clones of nonswitching lineages in the o-smooth phenotype. Our results demonstrate that cells in a high frequency mode of switching continuously undergo changes in the size of both the $\mathrm{R}$ homologues, but cells in a low frequency mode of switching and expressing the basic o-smooth phenotype do not undergo similar: changes.

We have found that the size of the $\mathrm{R}$ chromosomes can increase as well as decrease in cells undergoing high frequency phenotypic switching and that changes from large to small or small to large are progressive. Since $C$. albicans 'is thought to contain between 50 and $130 \mathrm{rDNA}$ repeats per cell (Iwaguchi et al., 1992), the alterations in size of the $\mathrm{R}$ chromosomes could be explained by unequal crossing-over between tandemly arrayed rDNA units. Unequal sister chromatid exchange during mitotic growth results in both deletions and duplications of rDNA repeat units in Saccharomyces cerevisiae (Szostak \& Wu, 1980). Alterations in the electrophoretic karyotypes of clinical isolates of $C$. albicans have been observed in recurrence patients, where isolates were obtained from the same patient in a longitudinal study (Asakura et al., 1991). With one exception, all band changes occurred within the chromosomes carrying the rDNA units. In another study, Iwaguchi et al. (1992) demonstrated that clonal variation of the $\mathrm{R}$ chromosomes occurred at frequencies as high as $10 \%$ of progeny clones and that these variations were directly attributed to the number of $\mathrm{rDNA}$ repeats per homologue. Therefore, alterations in the size of the $\mathrm{R}$ chromosomes appear to be common to $C$. albicans. However, it is important to note that in no study has there been a direct correlation between a colony morphology, a specific change in phenotype, and a specific chromosomal rearrangement (Suzuki et al., 1989; Rustchenko-Bulgac $e t$ al., 1990; Rustchenko-Bulgac, 1991; Iwaguchi et al., 1992; Rustchenko-Bulgac \& Howard, 1993).

The correlation between high frequency switching and the instability of the $\mathrm{R}$ chromosomes in C. albicans strain $3153 \mathrm{~A}$ shares an interesting similarity with the phenotype of particular mutations of the silent information regulator (SIR) genes of S. cerevisiae (Laurenson \& Rine, 1992). A mutation in the SIR 2 gene not only results in expression of the normally silent mating type loci $H M L$ and $H M R$, but also in a corresponding increase in intrachromosomal recombination between ribosomal cistrons (Gottleib \& Esposito, 1989). We are, therefore, pursuing the idea that the transition from a low to high frequency mode of switching in strain 3153A involves a change in the gene silencing machinery and chromatin structure.

\section{ACKNOWLEDGEMENTS}

The authors are indebted to Dr T. Srikantha for providing the rDNA probe. The study was supported by Public Health Service Grants AI 2392 and DE 10758 from the National Institutes of Health.

\section{REFERENCES}

Anderson, U., Srikantha, T., Morrow, B., Miyasaki, S. H., White, T. C., Agabian, N., Schmid, J. \& Soll, D. R. (1993). Characterization and partial nucleotide sequence of the DNA fingerprinting probe Ca3 of Candida albicans. J Clin Microbiol 31, 1472-1480.

Asakura, K., Iwaguchi, S.-I., Homma, M., Sukori, T., Higashide, K. \& Tanaka, K. (1991). Electrophoretic karyotypes of clinically isolated yeasts of Candida albicans and C. glabrata. J Gen Microbiol 137, 2531-2538.

Bedell, G. W. \& Soll, D. R. (1979). Effects of low concentrations of zinc on the growth and dimorphism of Candida albicans: evidence for zinc-resistant and sensitive pathways for mycelium formation. Infect Immun 26, 348-354.

Gottleib, S. \& Esposito, S. (1989). A new role for a yeast transcriptional silencer gene, SIR 2 , in regulation of recombination in ribosomal DNA. Cell 56, 771-776.

Iwaguchi, S.-I., Homma, M. \& Taraka, K. (1992). Clonal variation of chromosome size derived from the rDNA cluster region in Candida albicans. J Gen Microbiol 138, 1177--1184.

Lasker, B. A., Carle, G. F., Kobayashi, G. S. \& Medoff, G. (1989). Comparison of the separation of Candida albicans chromosome-sized DNA by pulsed-field gel electrophoresis techniques. Nucleic Acids Res 17, 3783-3793.

Laurenson, P. \& Rine, J. (1992). Silencers, silencing, and heritable transcriptional states. Microbiol Rev 56, 543-560.

Lee, K. L., Buckley, H. R. \& Campbell, C. C. (1975). An amino acid liquid synthetic medium for development of mycelial and yeast forms of Candida. Sabouraudia 13, 148-153.

Magee, B. B. \& Magee, P. T. (1987). Electrophoretic karyotypes and chromosome numbers in Candida species. J Gen Microbiol 133 425-430.

McEachern, M. J. \& Hicks, J. B. (1991). Dosage of the smallest chromosome affects both the yeast-hyphal transition and the white-opaque transition of Candida albicans WO-1. J Bacteriol 173, $7436-7442$.

Merz, W. G., Connelly, C. \& Hieter, P. (1988). Variation of electrophoretic karyotypes among clinical isolates of Candida albicans. J Clin Microbiol 26, 842-845.

Morrow, B., Anderson, J., Wilson, E. \& Soll, D. R. (1989). Bidirectional stimulation of the white-opaque transition of $C$ andida albicans by ultraviolet irradiation. J Gen Microbiol 135, 1201-1208.

Morrow, B., Srikantha, T. \& Soll, D. R. (1992). Transcription of the gene in a pepsinogen, PEP1, is regulated by white-opaque switching in Candida albicans. Mol Cell Biol 12, 2997-3005.

Morrow, B., Ramsey, H. \& Soll, D. R. (1994). Regulation of phase specific genes in the more general switching system of Candida albicans strain 3153A. J Med Vet Mycol (in press).

Pomes, R., Gil, C. \& Nombela, C. (1985). Genetic analysis of Candida albicans morphological mutants. J Gen Microbiol 131, 2107-2113.

Rikkerink, E. H. A., Magee, B. B. \& Magee, P. T. (1988). Opaque 
white phenotype transition: a programmed morphological transition in Candida albicans. J Bacteriol 170, 895-899.

Rustchenko-Bulgac, E.P. (1991). Variations of Candida albicans electrophoretic karyotypes. J Bacteriol 173, 6586-6596.

Rustchenko-Bulgac, E.P. \& Howard, D. H. (1993). Multiple chromosomal and phenotypic changes in spontaneous mutants of Candida albicans. J Gen Microbiol 139, 1195-1207.

Rustchenko-Bulgac, E. P., Sherman, F. \& Hicks, J. B. (1990). Chromosomal rearrangements associated with morphological mutants provide a means for genetic variation in Candida albicans. $J$ Bacteriol 172, 1276-1283.

Sadhu, C., McEachern, M. J., Rustchenko-Bulgac, E. P., Schmid, J., Soll, D. R. \& Hicks, J. B. (1991). Telomeric and dispersed repeat sequences in Candida yeasts and their use in strain identification. $J$ Bacteriol 173, 842-850.

Schmid, J., Voss, E. \& Soll, D. R. (1990). Computer-assisted methods for assessing Candida albicans strain relatedness by Southern blot hybridization with repetitive sequence Ca3. J Clin Microbiol 28, 1236-1243.

Slutsky, B., Buffo, J. \& Soll, D. R. (1985). High frequency switching of colony morphology in Candida albicans. Science 230, 666-669.

Slutsky, B., Staebell, M., Anderson, J., Risen, L., Pfaller, M. \& Soll, D. R. (1987). 'White-opaque transition': a second high-frequency switching system in Candida albicans. J Bacteriol 169, 189-197.

Soll, D. R. (1990). Dimorphism and high frequency switching in
Candida albicans. In The Genetics of Candida albicans, pp. 147-176. Edited by D. R. Kirsch, R. Kelly \& M. B. Kurtz. Boca Raton, FL: CRC Press.

Soll, D. R. (1992). High-frequency switching in Candida albicans. Clin Microbiol Rev 5, 183-203.

Soll, D. R. (1993). DNA fingerprinting of Candida albicans. J Mycol Med 3, 37-44.

Soll, D. R., Morrow, B. \& Srikantha, T. (1993a). High frequency switching in Candida albicans. Trends Genet 9, 61-65.

Soll, D. R., Morrow, B. \& Srikantha, T. (1993b). Switching and gene regulation in Candida albicans. In The Eukaryotic Microbial Genome, pp. 211-240. Edited by P. M. A. Broda, S. G. Oliver \& P. G. Sims. Cambridge: Cambridge University Press.

Suzuki, T., Kobayashi, I., Kanbe, T. \& Tanaka, K. (1989). High frequency variation in colony morphology and chromosome reorganization in the pathogenic yeast Candida albicans. J Gen Microbiol 135, 425-434.

Szostak, J. W. \& Wu, R. (1980). Unequal crossing over in the ribosomal DNA of Saccharomyces cerevisiae. Nature 284, 426-430.

Vollrath, D. \& Davis, R. W. (1987). Resolution of greater than 5 megabase pair DNA molecules by contour-clamped homogeneous electric fields. Nucleic Acids Res 15, 7865-7876.

Received 20 September 1993; revised 10 January 1994; accepted 27 January 1994. 